\title{
miR-24 promotes the proliferation, migration and invasion in human tongue squamous cell carcinoma by targeting FBXW7
}

\author{
JINGZHU ZHAO ${ }^{1}$, CHUANXIANG HU $^{1}$, JIADONG CHI ${ }^{1}$, JIANSEN LI $^{2}$, CHEN PENG $^{1}$, XINWEI YUN ${ }^{1}$, \\ DAPENG LI ${ }^{1}$, YANG YU ${ }^{1}$, YIGONG LI ${ }^{1}$, MING GAO $^{1}$ and XIANGQIAN ZHENG ${ }^{1}$ \\ Departments of ${ }^{1}$ Thyroid and Neck Tumor and ${ }^{2}$ Anesthesiology, Tianjin Medical University \\ Cancer Institute and Hospital, Key Laboratory of Cancer Prevention and Therapy, \\ Tianjin National Clinical Research Center for Cancer, Tianjin 300060, P.R. China
}

Received January 17, 2016; Accepted February 24, 2016

DOI: $10.3892 /$ or.2016.4891

\begin{abstract}
Recent studies suggest that aberrant expression of miR-24 is linked to various human cancers, including tongue squamous cell carcinoma (TSCC). F-box and WD-40 domain protein 7 (FBXW7), a tumor-suppressor gene, is responsible for the degradation of several proto-oncogenes. However, the function and mechanism of miR-24 and FBXW7 in TSCC remains unclear. In the present study, we found that miR-24 was increased in TSCC tissues and cell lines, and that upregulation of miR-24 was associated with advanced clinical stage and a shorter overall survival of TSCC patients. Inhibition of miR-24 significantly suppressed the proliferation, migration and invasion of TSCC cells in vitro. Furthermore, miR-24 repressed FBXW7 expression by directly binding to the 3-untranslated region of FBXW7. Moreover, the suppression of FBXW7 increased the proliferation, migration and invasion of TSCC cells, and the restoration of FBXW7 substantially attenuated the oncogenic effects of miR-24. In conclusion, our results demonstrated that upregulation of miR-24 was associated with tumor progression and poor prognosis in TSCC patients, and that overexpression of miR-24 was correlated with the proliferation, migration and invasion of TSCC cells in vitro, at least partially through regulation of its functional target FBXW7. Thus, miR-24 may serve as a novel potential biomarker for the prognosis of TSCC patients.
\end{abstract}

Correspondence to: Dr Xiangqian Zheng or Dr Ming Gao, Department of Thyroid and Neck Tumor, Tianjin Medical University Cancer Institute and Hospital, Key Laboratory of Cancer Prevention and Therapy, Tianjin National Clinical Research Center for Cancer, Huanhuxi Road, Ti-Yuan-Bei, Hexi, Tianjin 300060, P.R. China

E-mail: headandneck2007@aliyun.com

E-mail: headandneck15@aliyun.com

Key words: miR-24, FBXW7, tongue squamous cell carcinoma, proliferation, migration, invasion

\section{Introduction}

Oral squamous cell carcinoma (OSCC) is one of the leading malignancies worldwide $(1,2)$. Tongue squamous cell carcinoma (TSCC) is the most common type of oral cancer and is known for its high rate of proliferation and nodal metastasis (3). According to the American Cancer Society, an estimated 13,590 new cases of tongue cancer were diagnosed in 2013, accounting for approximately $30 \%$ of all oral cavity and pharynx cancers, with an estimated 2070 deaths (4). Even with recent advances in its treatment, the 5-year survival rate remains at $\sim 50 \%$ (5). Although attempts have been made to identify the molecular pathways that contribute to the carcinogenesis and progression of TSCC, the mechanisms underlying TSCC progression are poorly understood.

MicroRNAs (miRNAs) are small, endogenous, non-coding RNAs involved in post-transcriptional regulation of gene expression (6) and are involved in cell growth, development, differentiation, proliferation and apoptosis (7-10). Recent studies have demonstrated that miRNAs play a critical role in tumorigenesis $(11,12)$ and are aberrantly expressed in different cancer types, including TSCC (13-16).

F-box and WD-40 domain protein 7 (FBXW7) is an E3 ubiquitin ligase that regulates the stability of many proteins, including cyclin E1, c-Myc, NOTCH1, c-Jun and Mcl-1 (17-19). Mutations in FBXW7 usually disrupt 3 key residues in the substrate-binding domain and lead to increased stability of target proteins. Many of the FBXW7 targets are known oncogenes, thus these mutations could promote tumorigenesis through multiple pathways. Based on oncology, clinical and basic research, the reduced expression or loss of function of FBXW 7 has been frequently found in a variety of human cancers, with an overall mutation frequency of $6 \%(20)$. Clinically, low expression of FBXW7 in human solid tumors such as glioma, colorectal cancer and gastric cancer is associated with a poor prognosis (21-24).

Upregulation of miR-24 has been observed in a number of cancers $(25-27)$, including OSCC $(28,29)$. Our previous study demonstrated that frequent upregulation of miR-24 occurred in TSCC, and miR-24 induced cell survival and cisplatin resistance in vitro (29). In the present study, we further found that miR-24 was associated with a shorter overall survival of 
TSCC patients, and that inhibition of miR-24 significantly suppressed the proliferation, migration and invasion of TSCC cells in vitro. Moreover, we identified and verified that FBXW7 is a functional target of miR-24, and miR-24 confers its effects in part through targeting FBXW7, which in turn regulates its downstream genes, including c-Myc and c-Jun.

\section{Materials and methods}

Patients and TSCC tissues. Eighty-four paired TSCC and corresponding non-tumor control tissues were obtained from patients through surgical dissection at the Tianjin Cancer Hospital and Institute. This study was approved by the Ethics Committee of the Tianjin Cancer Hospital and Institute, and written informed consent was obtained from all patients.

Cell lines. Human tongue cancer cell lines (UM1, UM2, Ca127, SCC1, SCC15 and SCC25) were obtained from the American Type Culture Collection (ATCC; Manassas, VA, USA). The cells were cultured in Dulbecco's modified Eagle's medium (DMEM; Invitrogen), supplemented with $10 \%$ fetal bovine serum (FBS); all cell lines were maintained at $37^{\circ} \mathrm{C}$ in $5 \% \mathrm{CO}_{2}$, respectively.

RNA extraction and quantitative RT-PCR ( $q R T-P C R)$. TRIzol reagent (Invitrogen, Carlsbad, CA, USA) was used to isolate total RNAs from frozen tissues and TSCC cells according to the manufacturer's protocol. Expression of miRNAs was analyzed by mirVana qRT-PCR miRNA detection assay (Ambion) or TaqMan miRNA assay (Applied Biosystems) according to the manufacturer's protocol. TaqMan microRNA assays for U6 was used to normalize the relative abundance of miRNA in the tissues and cell lines.

Cell transfection. Cell transfection with anti-miR-NC, anti-miR-24, si-NC or si-FBXW7 was conducted by using Lipofectamine 2000 (Invitrogen) according to the manufacturer's instructions.

Specific miRNA-expression plasmids were created by PCR amplification of human genomic DNA fragments. The primers were: miR-24 sense, TGGAAGTTTGAGAGTTGAG CCG and antisense, ACCTTCAAACTCTCAACTCGGC. The PCR products (pre-miRNAs) were cloned into the pcDNA3.1/V5-His-TOPO expression vector (Invitrogen) and confirmed by DNA sequencing. The expression of miRNA was carried out by transfection of the plasmid into the cells using Lipofectamine 2000.

Cell proliferation assay. SCC15, SCC25 and Cal27 cells were seeded onto 96 -well plates $\left(2 \times 10^{4}\right.$ cells/well $)$, respectively. The cells were transfected with anti-miR-NC, anti-miR-24, miR-NC, miR-24, si-NC, si-FBXW7, empty vector, FBXW7 vector or cotransfected with the miR-24 and FBXW7 vectors, respectively. After $48 \mathrm{~h}$, cell proliferation was analyzed using Cell Counting Kit-8 (CCK-8; Beyotime) according to the manufacturer's protocol.

Cell migration and invasion assays. SCC15, SCC25 and Cal27 cells were cultured and transfected with anti-miR-NC, anti-miR-24, miR-NC, miR-24, si-NC, si-FBXW7, empty vector, FBXW7 vector or co-transfected with miR-24 and
FBXW7 vectors, respectively. Cell migration and invasion assays were performed by using Transwell chambers (Millipore, Billerica, MA, USA). Filters for the invasion assay were precoated with Matrigel (BD Biosciences, Franklin Lakes, NJ, USA) in the upper compartment. Cells $\left(5 \times 10^{5}\right)$ were seeded in the upper compartment, and the lower compartment was filled with DMEM supplemented with $10 \%$ FBS. After $24 \mathrm{~h}$ of incubation, the migratory and invasive cells adherent to the undersurface of the filters were fixed, stained with crystal violet and four random fields were counted for each group.

Luciferase reporter assays. A FBXW7 3'UTR luciferase reporter was created. Briefly, complementary 48-mer DNA oligonucleotides containing the putative binding site of miR-24 were synthesized with flanking $M l u I$ and HindIII restriction enzyme digestion sites. The pmirGLO Dual-Luciferase miRNA Target expression vector (Promega, Madison, WI, USA; E1910 expressing both firefly and Renilla) was employed to construct the luciferase reporter vector. Double-stranded oligonucleotides corresponding to the wild-type (wt-3'UTR) or mutant (mu-3'UTR) miR-24 binding site in the FBXW7 3'UTR were synthesized (Sangon Biotech) and ligated between the XolI and SbfI restriction sites of the reporter plasmid pmirGLO (Promega). The sequences of inserted fragments were confirmed by sequencing.

HEK293T cells were transfected with wild-type pmirFBXW7-3'UTR or mutant pmir-FBXW7-3'UTR, and the Renilla luciferase control vector (pRL-TK; Promega) using Lipofectamine 2000, and then the cells were transfected with miR-NC or miR-24, respectively. Luciferase activities were measured at $24 \mathrm{~h}$ after transfection by using a dual-luciferase assay kit (E1330; Promega). Firefly luciferase activities were normalized to Renilla luciferase activities.

Western blot analysis. To isolate the proteins, cells harvested from 6-well plates were washed once in phosphate-buffered saline and lysed in lysis buffer. Protein lysates were separated on $10 \%$ Tris-glycine polyacrylamide gel and then transferred to a polyvinylidene fluoride (PVDF) membrane (Zhongshan Biotechnology Co. Ltd., Beijing, China). Then, the membrane was incubated with monoclonal antibodies against FBW7, c-Myc and c-Jun, followed by incubation with HRP (horseradish peroxidase)-labeled goat-anti-mouse IgG (Santa Cruz Biotechnology, Inc., TX, USA). $\beta$-actin was used as a protein loading control. The intensity of the protein was quantified with the Quantity software LANE-1D analyzer (SmartGel; Sage Creation).

Statistical analysis. Data were analyzed using the Statistical Package for the Social Sciences (SPSS, Chicago, IL, USA), version 17.0. All values are presented as mean \pm standard deviation (SD). One-way ANOVA and two-tail Student's t-test were used to analyze the results. A p-value $<0.05$ was considered to indicate statistical significance.

\section{Results}

miR-24 is significantly increased in TSCC tissues and cell lines and upregulation of miR-24 correlates with poor patient prognosis. To detect whether there was any difference in the 

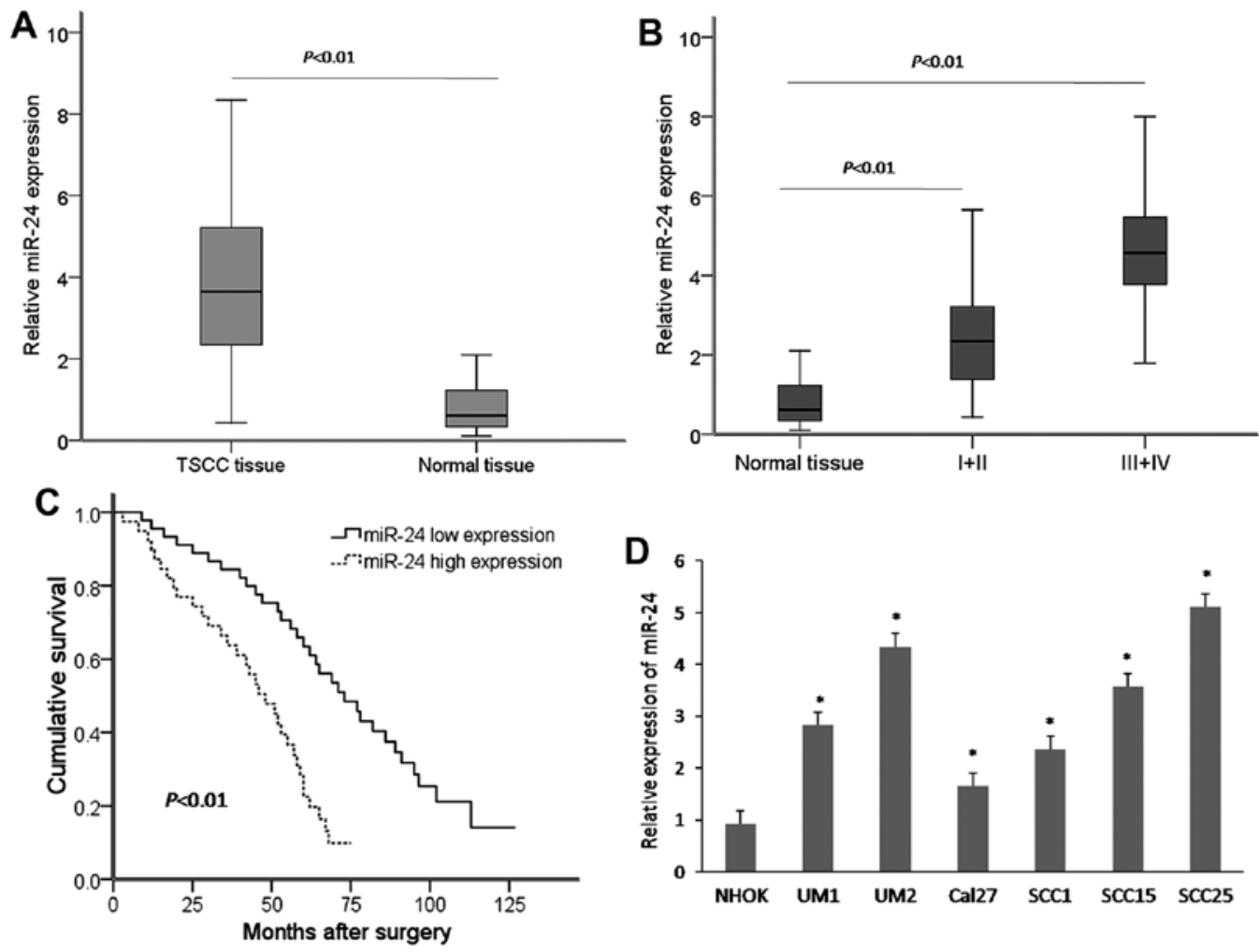

Figure 1. miR-24 is increased in TSCC tissues and cell lines and is correlated with poor prognosis in patients with TSCC. (A) Expression of miR-24 in 84 TSCC tissues and matched normal tissues was detected by qRT-PCR. (B) Expression of miR-24 was positively correlation with clinical stage. (C) Overall survival curves of miR-24 in patients with TSCC for two groups defined by low and high expression of miR-24. (D) Expression of miR-24 in normal oral keratinocyte cell culture (NHOK) and six TSCC cell lines was detected by qRT-PCR. Expression of miR-24 was normalized to U6. All assays were performed in duplicates. ${ }^{*} \mathrm{P}<0.01$ compared with the control.

miR-24 expression level between TSCC tissues and adjacent non-tumor tissues, a total of 84 paired TSCC tissues and adjacent non-tumor tissue samples were subjected to qRT-PCR. Compared with the matched non-tumor tissues, the expression level of miR-24 was significantly increased in the TSCC tissues (Fig. 1A). Furthermore, the association of miR-24 with clinicopathologic factors of the TSCC cases was examined. miR-24 overexpression was associated with advanced clinical stage (Fig. 1B). Kaplan-Meier survival analysis showed that the overall survival of TSCC patients with high miR-24 expression was significantly shorter than that with low miR-24 expression (Fig. 1C). In additional, miR-24 expression was significantly higher in six TSCC cell lines (UM1, UM2, Cal27, SCC1, SCC15 and SCC25) compared with that in the nontumorigenic cells (NHOK) (Fig. 1D).

miR-24 regulates the proliferation, migration and invasion of TSCC cells in vitro. To determine whether miR-24 promotes the proliferation, migration and invasion potential of TSCC cells, we carried out experiments using CCK-8, migration and invasion assays. Briefly, SCC15 and SCC25 cells were transfected with anti-miR-NC or anti-miR-24 for $24 \mathrm{~h}$, and Cal27 cells were transfected with miR-NC or miR-24 for $24 \mathrm{~h}$, followed by analysis of the proliferation, migration and invasion of the cells, respectively. The data showed that expression of miR-24 in the SCC15 and SCC25 cells transfected with antimiR-24 was significantly decreased by qRT-PCR (Fig. 2A), whereas expression of miR-24 was markedly increased in the Cal27 cells (Fig. 2E). Furthermore, transfection of anti-
miR-24 significantly suppressed the proliferation, migration and invasion of the SCC15 and SCC25 cells compared to these parameters in the control group (Fig. 2B-D), whereas miR-24 markedly enhanced the proliferation, migration and invasion of Cal27 cells (Fig. 2F-H). Together, miR-24 regulates the proliferation, migration and invasion of TSCC cells.

FBXW7 is a direct target of miR-24 and is negatively regulated by miR-24. To explore the molecular mechanism of miR-24 in TSCC, we searched for candidate target genes using bioinformatics algorithms of TargetScan. We focused on tumor-suppressor gene FBXW7, which has one potential miR-24 binding site within its 3'UTR.

To identify whether FBXW7 is a target of miR-24, we constructed vectors containing the wild-type 3'UTR or mutant 3'UTR of FBXW7 mRNA, which was individually fused directly downstream of the firefly luciferase gene (Fig. 3A). For the luciferase assays, the wild-type or mutant vector was co-transfected into HEK293T cells with miR-NC or miR-24. Luciferase reporter assay showed that miR-24 could inhibit the luciferase activity of the wild-type 3'UTR of the FBXW7 reporter vector but not the mutant 3'UTR (Fig. 3B). Furthermore, we confirmed that overexpression of miR-24 could decrease FBXW7 mRNA levels, whereas inhibition of miR-24 increased the FBXW7 mRNA levels (Fig. 3C). Moreover, FBXW7 protein abundance was also negatively regulated by miR-24 (Fig. 3D). Together, miR-24 downregulated FBXW7 expression by directly targeting the 3'UTR of FBXW7. 

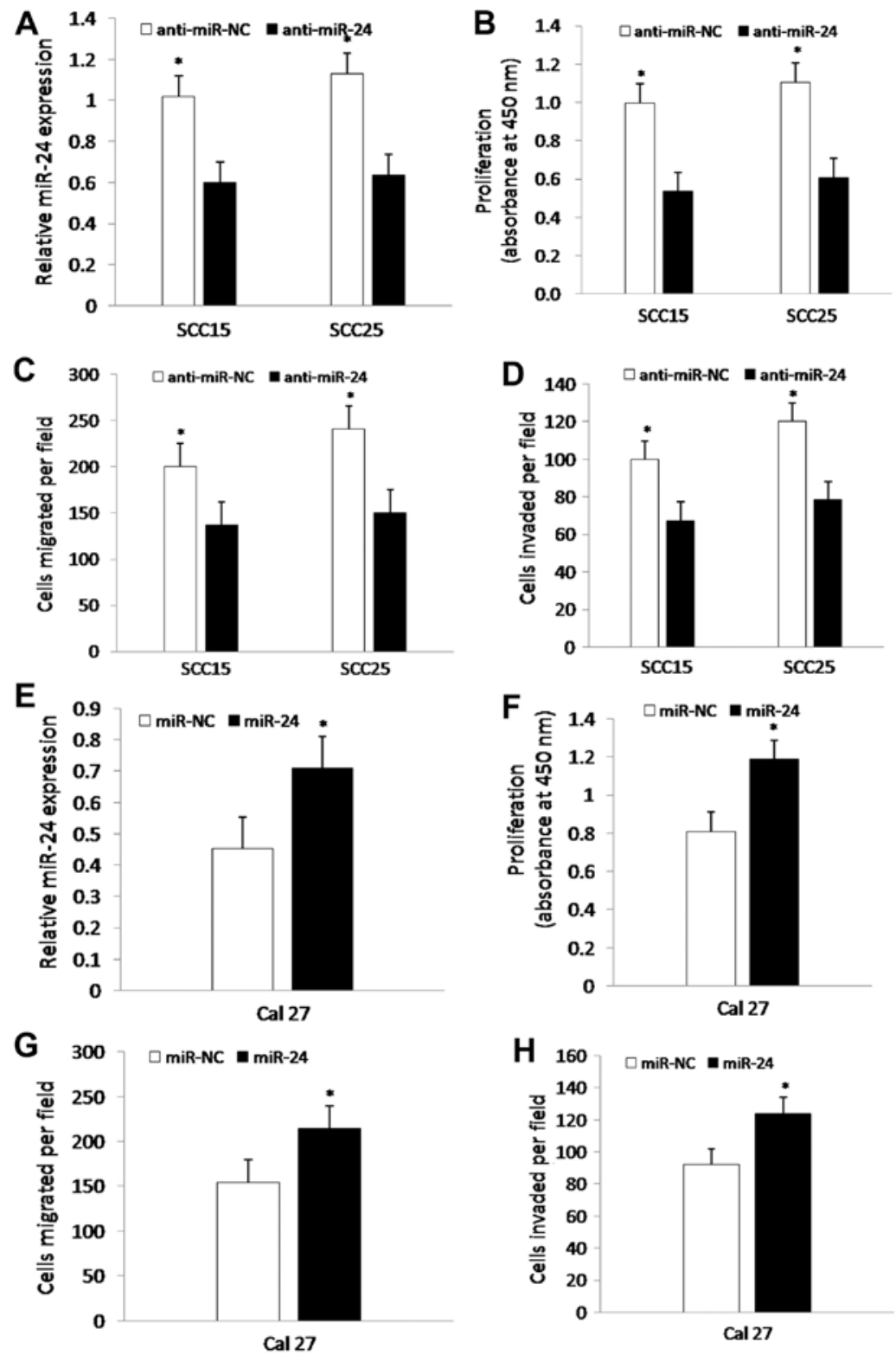

Figure 2. Effect of miR-24 on proliferation, migration and invasion of TSCC cells in vitro. SCC15 and SCC25 cells were transfected with anti-miR-NC or antimiR-24 for $24 \mathrm{~h}$, respectively. Cal27 cells were transfected with miR-NC or miR-24 for $24 \mathrm{~h}$. (A) Expression of miR-24 in SCC15 and SCC25 cells transfected with anti-miR-NC or anti-miR-24 was detected by qRT-PCR. (E) Expression of miR-24 in Cal27 cells transfected with miR-NC or miR-24 was detected by qRT-PCR. After transfection, the cells were used to perform the proliferation (B and F), migration (C and $\mathrm{G}$ ) and invasion (D and $\mathrm{H})$ assays. All assays were repeated in duplicates. " $\mathrm{P}<0.01$, compared with the control.

miR-24 promotes TSCC cell growth and motility by targeting FBXW7. To investigate the effect of FBXW7 on tumorigenicity, we designed experiments in which the SCC15 and SCC25 cells were transfected with si-NC, si-FBXW7, empty vector and FBXW7 vector. The results showed that the growth and motility of the SCC15 and SCC25 cells were markedly promoted or suppressed after transfection with si-FBXW7 or the FBXW7 vector, respectively (Fig. 4A-C), suggesting that FBXW7 suppresses the proliferation, migration and invasion of TSCC cells. To further assess whether the effect of miR-24 on tumorigenicity was via targeting FBXW7, we carried out functional experiments. Briefly, the SCC15 and SCC25 cells were transfected with miR-NC, miR-24 or co-transfected with miR-24 and FBXW7. The growth and motility of the SCC15 and SCC 25 cells were significantly enhanced after transfection with miR-24 compared with the control group, whereas the restoration of FBXW7 antagonized miR-24 in regards to the migration, invasion and proliferation (Fig. 4D-F). Furthermore, western blotting showed that FBXW7 expression was markedly decreased in the TSCC cells after transfection with miR-24, and was restored when the TSCC cells were co-transfected with miR-24 and FBXW7, and subsequently inversely regulated its downstream targets c-Myc and c-Jun (Fig. 4G). Together, these findings suggest that miR-24 promotes the proliferation, migration and invasion, at least partially by targeting FBXW7.

\section{Discussion}

The development of TSCC is a multistep process involving multiple factors. Great efforts have been made to understand 


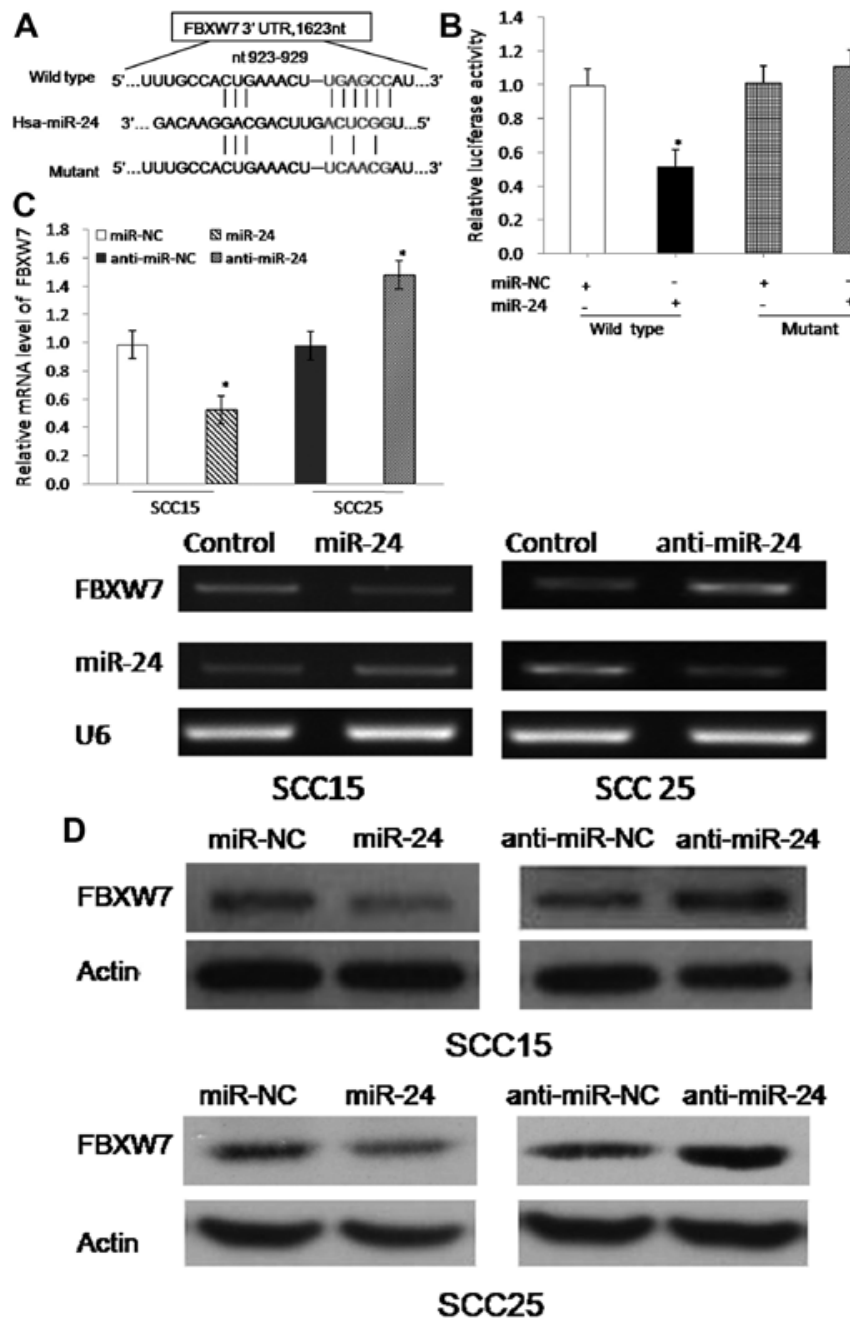

Figure 3. FBXW7 is a direct target of miR-24. (A) The wild-type or mutant binding site of the FBXW7-3'-untranslated region (3'UTR) to miR-24. (B) The HEKT293 cells were transfected with luciferase vectors containing wild-type or mutant FBXW7-3'UTR and miR-NC or miR-24, respectively. Relative luciferase activity as detected by luciferase reporter assay. mRNA expression of FBXW7 in SCC15 and SCC25 cells after transfection with miR-NC, miR-24, anti-miR-NC or anti-miR-24 was detected by qRT-PCR (C, upper panel) and RT-PCR (C, lower panel) assays. (D) Western blotting was used to detect the protein levels of FBXW7 in SCC15 and SCC25 cells after transfection with miR-NC, miR-24, anti-miR-NC or anti-miR-24. $\beta$-actin was used as control. The assays were repeated in duplicates. ${ }^{*} \mathrm{P}<0.01$ compared with the control group.

the principles of molecular changes during the occurrence of this malignancy. To date, accumulating evidence demonstrates that multiple miRNAs are involved in TSCC development and progression $(13,15,16)$. miR-24 has been found to serve as an oncogene in several types of cancers, including breast carcinoma, glioma, oral carcinoma, acute myeloid leukemia and squamous cell carcinoma (30-33). Recently, it was reported that this miR-24 had a close association with $\operatorname{TSCC}(28,29)$, suggesting that it may serve as a potential biomarker for the diagnosis and prognosis of TSCC. However, the mechanism of miR-24 in TSCC is not entirely clear.

In our present study, we found that miR-24 was significantly overexpressed in human TSCC tissues and cell lines compared with the control groups, and that upregulation of miR-24 was associated with a shorter overall survival of TSCC patients. In agreement with our findings, previous reports have shown that miR-24 is overexpressed in many other solid tumors, and is related to tumor progression and prognosis. It is known that microRNAs function primarily by negatively regulating the expression of their target genes. And recent studies have demonstrated some specific targets of miR-24 in several tumors, such as the sex determining region Y (SRY)-box 7 (SOX7) (34), nuclear apoptosis-inducing factor 1 (NAIF1) (35) and dead end 1 (DND1) (14). In addition, our previous study confirmed that miR-24 induced cell survival and cisplatin resistance primarily through targeting the PTEN/Akt pathway in TSCC cell lines (29). We further confirmed that FBXW7 is a direct target of miR-24. And our functional analysis showed that miR-24-induced loss of FBXW7 enhanced TSCC cell proliferation, migration and invasion, and that miR-24 expression responds to alterations in the c-Myc and c-Jun protein levels, which were regulated by the FBXW7 pathway. These findings suggest that overexpression of miR-24 promotes the proliferation, migration and invasion of TSCC cells in vitro, possibly due to suppression of the function of FBXW7.

FBXW7 regulates the stability of several substrates, which are involved in the regulation of apoptosis and cell proliferation, such as c-Myc, cyclin E, Notch1 and Mcl-1 (17,36-38). In addition, accumulating evidence shows that FBXW7 plays critical roles in tumorigenesis. To date, there are a few agents known to activate FBXW7, such as miR-27, miR-92, miR-25 

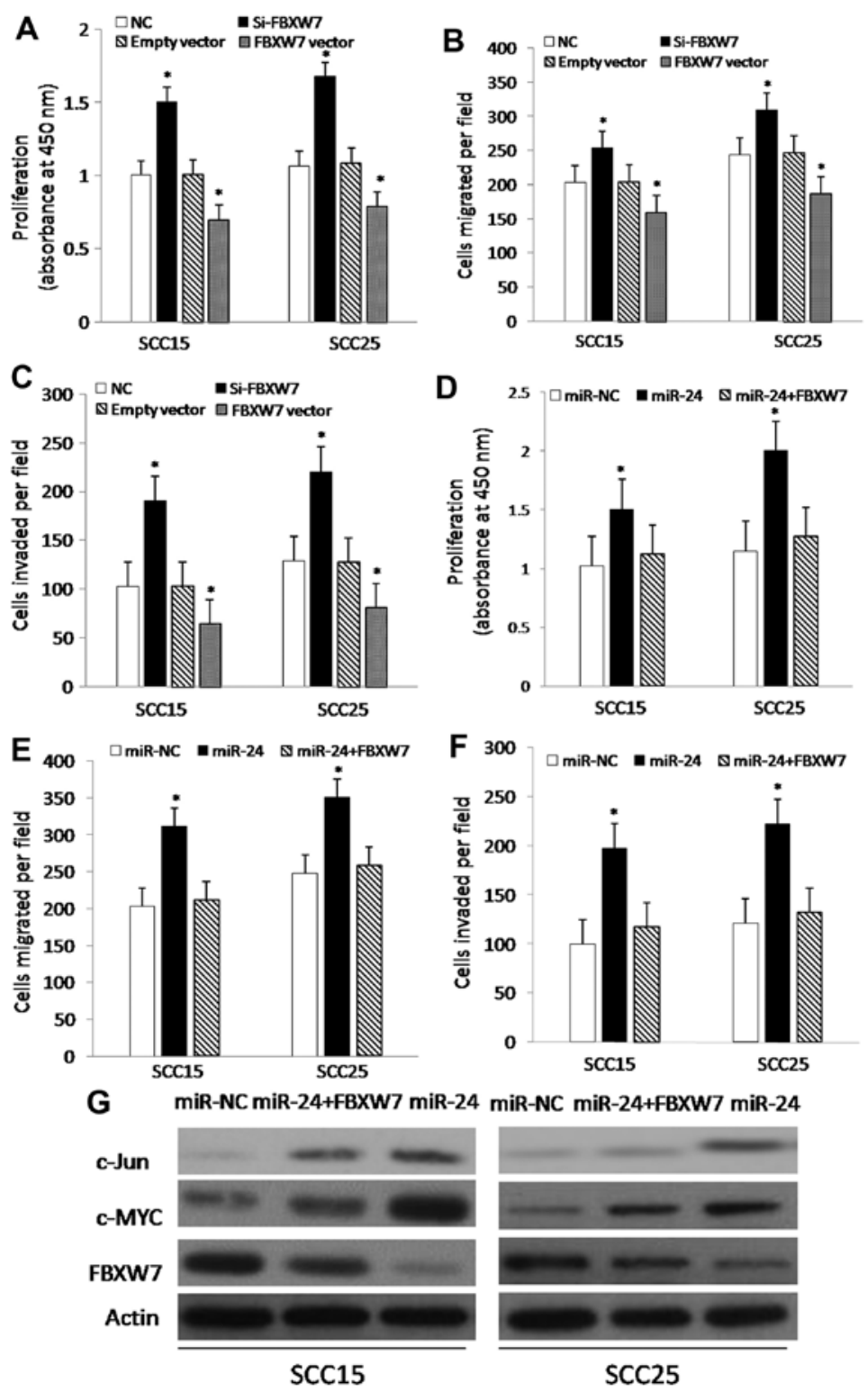

Figure 4. Suppression of FBXW7 promotes TSCC cell growth and motility and restoration of FBXW7 attenuates cell growth and motility. (A-C) Cell proliferation, migration and invasion ability assays of SCC15 and SCC25 cells following transfection with si-NC, si-FBXW7, empty vector, or FBXW7 vector, respectively. Histogram reveals the values of absorbance at $450 \mathrm{~nm}$ for proliferation (A). The assays were repeated in duplicates. (D-F) Cell proliferation, migration and invasion ability assays in SCC15 and SCC25 cells following transfection with miR-NC, miR-24, or co-transfection with miR-24 and FBXW7, respectively. Histogram reveals the values of absorbance at $450 \mathrm{~nm}$ for proliferation (D). The assays were repeated in duplicates. (G) Western blot assays show the protein expression of FBXW7, c-Myc and c-Jun in the SCC15 and SCC25 cells transfected with miR-NC, miR-24, or co-transfected with miR-24 and FBXW7, respectively. $\beta$-actin served as an internal control. Western blot analysis confirmed that miR-24 downregulated the expression of FBXW7 protein and inversely upgulated the downstream targets c-Myc and c-Jun. The assays were repeated in duplicates. ${ }^{*} \mathrm{P}<0.01$, compared with the control group.

and miR-223 in several cancers (39-41). Here, we confirmed that FBXW7 is a direct target of miR-24. Furthermore, a decrease in the expression of FBXW7 resulting from the overexpression of miR-24 gave rise to abnormal accumulation of c-Myc and c-Jun proteins. Our results suggest that miR-24 may exert its oncogenic function partially through elevating c-Myc and c-Jun expression. In addition, studies in mice show that FBXW7 inhibition eliminates CML by increasing Myc abundance (42). Kurashige indicates that high expression of miR-223 had a significant adverse impact on the survival of ESCC patients through repression of the function of FBXW7 (43). Taking these results together with our findings, we surmise that miR-24-induced loss of FBXW7 may be a promising treatment target for TSCC due to its ability to reduce the levels of several oncoproteins.

In summary, the present study indicated that deregulation of miR-24 is a frequent event in TSCC in vitro, and that miR-24 promoted the growth and motility of TSCC cell lines, at least partially by targeting FBXW7. We hope that our investigation can facilitate further exploration of the molecular mechanisms of miR-24 in TSCC.

\section{Acknowledgements}

The present study was partially supported by grants from the National Natural Science Foundation of China (grant nos. 
$81402392,81502322)$ and the Tianjin Municipal Science and Technology Project (grant no. 15JCQNJC12800).

\section{References}

1. Marcus B, Arenberg D, Lee J, Kleer C, Chepeha DB, Schmalbach CE, Islam M, Paul S, Pan Q, Hanash S, et al: Prognostic factors in oral cavity and oropharyngeal squamous cell carcinoma. Cancer 101: 2779-2787, 2004.

2. Chang KW, Liu CJ, Chu TH, Cheng HW, Hung PS, Hu WY and Lin SC: Association between high miR-211 microRNA expression and the poor prognosis of oral carcinoma. J Dent Res 87: 1063-1068,2008.

3. Siegel R, Naishadham D and Jemal A: Cancer statistics, 2012. CA Cancer J Clin 62: 10-29, 2012.

4. Siegel R, Naishadham D and Jemal A: Cancer statistics, 2013. CA Cancer J Clin 63: 11-30, 2013.

5. Sano D and Myers JN: Metastasis of squamous cell carcinoma of the oral tongue. Cancer Metastasis Rev 26: 645-662, 2007.

6. Gao B, Gao K, Li L, Huang Z and Lin L: miR-184 functions as an oncogenic regulator in hepatocellular carcinoma (HCC). Biomed Pharmacother 68: 143-148, 2014.

7. Zhang C: Novel functions for small RNA molecules. Curr Opin Mol Ther 11: 641-651, 2009.

8. Lujambio A and Lowe SW: The microcosmos of cancer. Nature 482: 347-355, 2012

9. Eulalio A, Mano M, Dal Ferro M,Zentilin L, Sinagra G, Zacchigna $\mathrm{S}$ and Giacca M: Functional screening identifies miRNAs inducing cardiac regeneration. Nature 492: 376-381, 2012.

10. Swarbrick A, Woods SL, Shaw A, Balakrishnan A, Phua Y Nguyen A, Chanthery Y, Lim L, Ashton LJ, Judson RL, et al: miR-380-5p represses p53 to control cellular survival and is associated with poor outcome in MYCN-amplified neuroblastoma. Nat Med 16: 1134-1140, 2010.

11. He L, He X, Lowe SW and Hannon GJ: microRNAs join the p53 network - another piece in the tumour-suppression puzzle. Nat Rev Cancer 7: 819-822, 2007.

12. Esquela-Kerscher A and Slack FJ: Oncomirs - microRNAs with a role in cancer. Nat Rev Cancer 6: 259-269, 2006.

13. Wong TS, Liu XB, Wong BY, Ng RW, Yuen AP and Wei WI Mature miR-184 as potential oncogenic microRNA of squamous cell carcinoma of tongue. Clin Cancer Res 14: 2588-2592, 2008.

14. Liu X, Wang A, Heidbreder CE, Jiang L, Yu J, Kolokythas A, Huang L, Dai Y and Zhou X: MicroRNA-24 targeting RNA-binding protein DND1 in tongue squamous cell carcinoma. FEBS Lett 584: 4115-4120, 2010.

15. Jia LF, Wei SB, Gong K, Gan YH and Yu GY: Prognostic implications of micoRNA miR-195 expression in human tongue squamous cell carcinoma. PLoS One 8: e56634, 2013.

16. Li J, Huang H, Sun L, Yang M, Pan C, Chen W, Wu D, Lin Z, Zeng $\mathrm{C}$, Yao Y, et al: $\mathrm{miR}-21$ indicates poor prognosis in tongue squamous cell carcinomas as an apoptosis inhibitor. Clin Cancer Res 15: 3998-4008, 2009

17. Welcker M and Clurman BE: FBW7 ubiquitin ligase: A tumour suppressor at the crossroads of cell division, growth and differentiation. Nat Rev Cancer 8: 83-93, 2008.

18. Cheng Y and Li G: Role of the ubiquitin ligase Fbw7 in cancer progression. Cancer Metastasis Rev 31: 75-87, 2012.

19. Brandt Y, Mitchell T, Wu Y and Hartley RS: Developmental downregulation of Xenopus cyclin $\mathrm{E}$ is phosphorylation and nuclear import dependent and is mediated by ubiquitination. Dev Biol 355: 65-76, 2011.

20. Akhoondi S, Sun D, von der Lehr N, Apostolidou S, Klotz K, Maljukova A, Cepeda D, Fiegl H, Dafou D, Marth C, et al: FBXW7/hCDC4 is a general tumor suppressor in human cancer. Cancer Res 67: 9006-9012, 2007.

21. Hagedorn M, Delugin M, Abraldes I, Allain N, BelaudRotureau MA, Turmo M, Prigent C, Loiseau H, Bikfalvi A and Javerzat S: FBXW7/hCDC4 controls glioma cell proliferation in vitro and is a prognostic marker for survival in glioblastoma patients. Cell Div 2: 9, 2007.

22. Bredel M, Bredel C, Juric D, Harsh GR, Vogel H, Recht LD and Sikic BI: Functional network analysis reveals extended gliomagenesis pathway maps and three novel MYC-interacting genes in human gliomas. Cancer Res 65: 8679-8689, 2005.

23. Iwatsuki M, Mimori K, Ishii H, Yokobori T, Takatsuno Y, Sato T, Toh H, Onoyama I, Nakayama KI, Baba H, et al: Loss of FBXW7, a cell cycle regulating gene, in colorectal cancer: Clinical significance. Int J Cancer 126: 1828-1837, 2010.
24. Yokobori T, Mimori K, Iwatsuki M, Ishii H, Onoyama I, Fukagawa T, Kuwano H, Nakayama KI and Mori M: p53-Altered FBXW7 expression determines poor prognosis in gastric cancer cases. Cancer Res 69: 3788-3794, 2009.

25. Wang X, Tang S, Le SY, Lu R, Rader JS, Meyers C and Zheng ZM: Aberrant expression of oncogenic and tumor-suppressive microRNAs in cervical cancer is required for cancer cell growth. PLoS One 3: e2557, 2008.

26. Volinia S, Calin GA, Liu CG, Ambs S, Cimmino A, Petrocca F, Visone R, Iorio M, Roldo C, Ferracin M, et al: A microRNA expression signature of human solid tumors defines cancer gene targets. Proc Natl Acad Sci USA 103: 2257-2261, 2006.

27. Liu R, Zhang H, Wang X, Zhou L, Li H, Deng T, Qu Y, Duan J, Bai M, Ge S, et al: The miR-24-Bim pathway promotes tumor growth and angiogenesis in pancreatic carcinoma. Oncotarget 6: 43831-43842, 2015.

28. Lin SC, Liu CJ, Lin JA, Chiang WF, Hung PS and Chang KW: miR-24 up-regulation in oral carcinoma: Positive association from clinical and in vitro analysis. Oral Oncol 46: 204-208, 2010.

29. Zheng X, Li J, Peng C, Zhao J, Chi J, Meng X, Yun X, Li D, $\mathrm{Yu}$ Y, Gao M, et al: MicroRNA-24 induces cisplatin resistance by targeting PTEN in human tongue squamous cell carcinoma. Oral Oncol 51: 998-1003, 2015.

30. Du WW, Fang L, Li M, Yang X, Liang Y, Peng C, Qian W, O'Malley YQ, Askeland RW, Sugg SL, et al: MicroRNA miR-24 enhances tumor invasion and metastasis by targeting PTPN9 and PTPRF to promote EGF signaling. J Cell Sci 126: 1440-1453, 2013.

31. Chen L, Zhang A, Li Y, Zhang K, Han L, Du W, Yan W, Li R, Wang Y, Wang K, et al: miR-24 regulates the proliferation and invasion of glioma by ST7L via $\beta$-catenin/Tcf- 4 signaling. Cancer Lett 329: 174-180, 2013.

32. Zaidi SK, Dowdy CR, van Wijnen AJ, Lian JB, Raza A, Stein JL, Croce CM and Stein GS: Altered Runx1 subnuclear targeting enhances myeloid cell proliferation and blocks differentiation by activating a miR-24/MKP-7/MAPK network. Cancer Res 69: 8249-8255, 2009

33. Papadimitriou E, Vasilaki E, Vorvis C, Iliopoulos D, Moustakas A, Kardassis D and Stournaras C: Differential regulation of the two RhoA-specific GEF isoforms Net1/Net1A by TGF- $\beta$ and miR-24: Role in epithelial-to-mesenchymal transition. Oncogene 31: 2862-2875, 2012

34. Ma Y, She XG, Ming YZ and Wan QQ: miR-24 promotes the proliferation and invasion of HCC cells by targeting SOX7. Tumour Biol 35: 10731-10736, 2014.

35. Zhao G, Liu L, Zhao T, Jin S, Jiang S, Cao S, Han J, Xin Y, Dong Q, Liu X, et al: Upregulation of miR-24 promotes cell proliferation by targeting NAIF1 in non-small cell lung cancer. Tumour Biol 36: 3693-3701, 2015.

36. Koepp DM, Schaefer LK, Ye X, Keyomarsi K, Chu C, Harper JW and Elledge SJ: Phosphorylation-dependent ubiquitination of cyclin E by the SCFFbw7 ubiquitin ligase. Science 294: 173-177, 2001.

37. Wu G, Lyapina S, Das I, Li J, Gurney M, Pauley A, Chui I, Deshaies RJ and Kitajewski J: SEL-10 is an inhibitor of notch signaling that targets notch for ubiquitin-mediated protein degradation. Mol Cell Biol 21: 7403-7415, 2001.

38. Inuzuka H, Shaik S, Onoyama I, Gao D, Tseng A, Maser RS, Zhai B, Wan L, Gutierrez A, Lau AW, et al: SCF(FBW7) regulates cellular apoptosis by targeting MCL1 for ubiquitylation and destruction. Nature 471: 104-109, 2011.

39. Olive V1, Sabio E, Bennett MJ, De Jong CS, Biton A, McGann JC Greaney SK, Sodir NM, Zhou AY, Balakrishnan A, et al: A component of the mir-17-92 polycistronic oncomir promotes oncogene-dependent apoptosis. Elife 2: e00822, 2013.

40. Wang L, Ye X, Liu Y, Wei W and Wang Z: Aberrant regulation of FBW7 in cancer. Oncotarget 5: 2000-2015, 2014.

41. Gong J, Cui Z, Li L, Ma Q, Wang Q, Gao Y and Sun H: MicroRNA-25 promotes gastric cancer proliferation, invasion, and migration by directly targeting F-box and WD-40 domain protein 7, FBXW7. Tumour Biol 36: 7831-7840, 2015.

42. Reavie L, Buckley SM, Loizou E, Takeishi S, Aranda-Orgilles B Ndiaye-Lobry D, Abdel-Wahab O, Ibrahim S, Nakayama KI and Aifantis I: Regulation of c-Myc ubiquitination controls chronic myelogenous leukemia initiation and progression. Cancer Cell 23: 362-375, 2013.

43. Kurashige J, Watanabe M, Iwatsuki M, Kinoshita K, Saito S, Hiyoshi Y, Kamohara H, Baba Y, Mimori K and Baba H: Overexpression of microRNA-223 regulates the ubiquitin ligase FBXW7 in oesophageal squamous cell carcinoma. Br J Cancer 106: 182-188, 2012. 